Methods Website metrics data were analysed for each unique user to determine geographic location and referral to the site. Information on the number of emails sent, for which STIs, and self-reported reasons for sending were collected from users who sent emails.

Results Launched in September 2012 without promotion, STCK has had 3,972 unique visitors. Most (77.5\%) visitors have been from the United States, almost half from California (29.6\%) and Washington DC (17.7\%). Most (62.3\%) first-time visitors found the website by typing its URL, $25.5 \%$ through Google, and less than $4 \%$ through any other single source. 30 visitors have sent anonymous emails to a total of 56 recipients. Most emails (76.9\%) were sent for unknown reasons, $10.6 \%$ were sent by users to themselves, $8.9 \%$ to partners, $1.3 \%$ as misuse, and $1.3 \%$ to a friend who was thought to be at risk for STIs. Most emails (57.9\%) were sent for more than one STI, $26.3 \%$ for chlamydia alone, and $15.8 \%$ for gonorrhoea alone.

Conclusion Without active promotion through clinics or online search optimization, very few visitors to STCK have used the website for anonymous partner notification or for misuse. Anonymous partner notification websites cannot assume that all emails sent through their service are for partner notification purposes. The uptake of STCK among clinic populations or following search engine optimization remains to be seen in the ongoing programme. Active promotion will be studied in the future.

\section{P5.005 STABLE RELATIONSHIP: BARRIER TO PARTNER MANAGEMENT OF SEXUALLY TRANSMITTED INFECTIONS}

doi:10.1136/sextrans-2013-051184.1052

${ }^{1}$ T Agyarko-Poku, ${ }^{2} Y$ Adu Sarkodie, ${ }^{3} \mathrm{~L}$ Atakorah-Yeboah, ${ }^{4} \mathrm{~S}$ Wambugu. ${ }^{1}$ Suntreso Government Hospital, Ghana Health Services, Kumasi, Ghana; ${ }^{2}$ School of Medical Sciences, Kwame Nkrumah University of Science and Technology, Kumasi, Ghana; ${ }^{3}$ Catholic University College, Fiapre Ghana, Sunyani, Ghana; ${ }^{4}$ FHI360 Ghana, Accra, Ghana

Background Effective partner involvement in management of sexually transmitted infections (STIs) remains one of the major pillars of efficient management of STIs. In clinical practise in Ghana, this has not been very easy to achieve as it behoves on the index patient to bring partners in to the health service provider. This is compounded by the fact that there are no laws to compel practitioner to enforce partner notification and reporting. This study investigates the relationship between the success of partner notification and marital status among patients attending Suntreso STI clinic in Kumasi, Ghana.

Methods Clinical records of Two thousand seven hundred and forty six (2,746; Male-37.2\%, 1022/2746; Female- 62.8\%, 1724/2746) patients who sought STI treatment between January 2010 and December 2011 were reviewed. Marital status, gender, notice of notification, response of partner of index patient and diagnosis were recorded. Data was analysed using SPSS

Results Of the 2,746 patients, $34.7 \%$ were married, $35.3 \%$ were single, $17.1 \%$ cohabitating and $12.9 \%$ separated/divorced. Partners of 61.3\% (1683/2746: Male- 29.0\%, 488/1683; Female-71.0\%, 1,195) of the index patients responded to the call. Those who responded, were made up of $19.1 \%$ (182/953) of the married persons, $58.9 \%(571 / 696)$ of single, $71.1 \%(334 / 470)$ cohabitating and $44.6 \%(157 / 354)$ separated/divorced.

Conclusion Married couples in relatively stable relationships had lower partner reporting rate $(19.1 \%)$ compared with those in other relations. Married males were more reluctant to report than female counterparts. Misunderstanding and possibility of mistrust among partners may have contributed to lower rates of partner reporting. Higher reporting rate among partners in cohabitating relationships may relate to the feeling of freedom and the ability to easily disengage from the relationship should strife arise

\section{P5.006 ROLE OF THE COCHRANE REVIEW GROUP OF SEXUALLY TRANSMITTED INFECTIONS AND THE GLOBAL PARTICIPATION IN THE DEVELOPMENT OF SYSTEMATIC REVIEWS}

doi:10.1136/sextrans-2013-051184.1053

${ }^{1} \mathrm{M}$ Torres, ${ }^{2} \mathrm{M}$ Diaz, ${ }^{3} \mathrm{C}$ Farquhar, ${ }^{4} \mathrm{H}$ Gaitán Duarte. ${ }^{1}$ Managing Editor. STI Cochrane Review Group, Bogota, Colombia; ${ }^{2} S T I$ Cochrane Review Group, Bogota, Colombia; ${ }^{3}$ Department of Obstetrics and Gynecology, University of Auckland, Auckland, New Zealand; ${ }^{4}$ Clinical Research Institute, Universidad Nacional de Colombia, Bogota, Colombia

Sexually transmitted infections are a substantial cause of morbidity across the world, and as a WHO report showed in 2005, are particularly common in adolescents. Untreated, or under-treated STIs can lead to complications such as infertility, stillbirths and chronic pelvic infection.

It is therefore no surprise that identifying and treating STIs are a priority for all health systems. Ensuring that available treatments, both those prescribed and those available without prescription are subject to a rigorous analysis of their benefits and harms, and that this information is made widely available is essential for informed decision making. Cochrane Systematic Reviews bring together all the evidence, analyse it in a consistent rigorous and structured manner and give the evidence in a summarised way to clinicians, policy makers and consumers.

The Cochrane Database of Systematic Review (CDSR) forms the main part of The Cochrane Library. It now has over 5000 reviews. Its impact factor for 2011 is 5.715 . Globally, more than $50 \%$ of health professionals enjoy one click access, free at the point of use, and every 4 seconds someone views the full text of a Cochrane Review.

The STI Cochrane Review Group has been re-registered in the year 2011 and since 2012 we undertake the task of providing evidence about the effectiveness and safety of interventions which seek to modify behaviours that increase the risk of STI acquisition, to prevent STI, to guide the treatment of STI of the etiological approach or under the syndromic approach.

We want to show the recent work of the STI Group, our network of more than 100 contribuitors from around the world and invite anyone interested in the development of new reviews, new protocols or review updates to be part of the group as well as to participate in the role of consumers and peer referees. www.sticr. cochrane.org

\section{P5.007 ANNUAL CHLAMYDIA TRACHOMATIS AND NEISSERIA GONORRHOEA TESTING IN AN ENDEMIC SETTING: THE ROLE OF CLIENT AND HEALTH CENTRE CHARACTERISTICS}

doi:10.1136/sextrans-2013-051184.1054

1,2B Hengel, ${ }^{3,2} \mathrm{~J}$ Ward, ${ }^{2} \mathrm{H}$ Wand, ${ }^{4,5} \mathrm{~A}$ Rumbold, ${ }^{2} \mathrm{~J}$ Kaldor, ${ }^{2} \mathrm{R}$ Guy, on behalf of the STRIVE InvestigatorOperational Group. 'Apunipima Cape York Health Council, Bungalow, Queensland, Australia; ${ }^{2}$ Kirby Institute, University of New South UK, Sydney, Australia; ${ }^{3}$ Baker IDI Heart and Diabetes Institute, Alice Springs, Australia; ${ }^{4}$ Menzies School of Health Research, Darwin, Australia; ${ }^{5}$ University of Adelaide, Adelaide, Australia

Background Chlamydia trachomatis (CT) and Neisseria gonorrhoea (NG) are endemic in many remote Australian Aboriginal communities. Clinical guidelines in remote areas recommend annual sexual health testing generally in 15 - 35 year olds, yet little is known about the extent of annual testing in remote health services or factors that predict it.

Methods We used baseline 2009-2010 laboratory data from 67 remote Aboriginal communities in four regions participating in a cluster randomised trial aiming to improve sexual health service delivery. We defined and calculated annual testing as the percentage of individuals with an initial negative CT/NG test that had a repeat test at 12 months, +/- 3 months. Using logistic regression we determined client and health service factors associated with an annual test. 
Results Among 6318 females recorded as testing negative for CT/ NG, 24\% had an annual test (highest in 16-19 year olds at 26\%), compared with $15 \%$ of 4241 males (highest in 20-24 year olds at $19 \%)$. Factors independently and significantly $(p<0.05)$ associated with annual testing were female gender (adjusted odds ratio (AOR): 1.7, 95\% CI: $1.5-1.9$ ), age $20-24$ years (AOR:1.5, 95\% CI: $1.2-1.9$ ), services with $>90 \%$ of clients

Aboriginal (AOR:1.4.95\% CI: 1.2-1.6), services having an electronic medical record system (AOR:1.7.95\% CI: 1.4-2.1), services using a centralised recall system (AOR:1.3.95\% CI: 1.1-1.6), services being community controlled (health centre governance controlled by a local Aboriginal board) (AOR:1.4, 95\% CI: 1.2-1.6) and Aboriginal health worker/s employed (AOR1.4.95\% CI: 1.0 -1.9).

Conclusions Our analysis identified both client and service related factors associated with higher levels of annual testing. Having Aboriginal staff members and systems which support patient recall were key factors identified for services that are amenable to change. While some factors may be indirect markers of other characteristics of the health centres or clients, these findings will help guide support to services in remote areas.

\section{P5.008 PERFORMANCE OF ANTENATAL SCREENING FOR HIV AND SYPHILIS IN EU/EEA, DURING 2006-2011: MAKING USE OF SURVEILLANCE DATA}

doi:10.1136/sextrans-2013-051184.1055

O Sfetcu, A Cleeve, G Likatavicius, G Spiteri, M van de Laar. ECDC, European Centre for Disease Prevention and Control, Stockholm, Sweden

Introduction Although majority of countries in the European Union (EU/EEA) offer antenatal screening for HIV and syphilis, mother-to-child transmission (MTCT) is still occurring. We aim to analyse the performance and effectiveness of antenatal screening programmes to strengthen these programmes across the EU/EEA. Methods Surveillance for HIV and syphilis, including congenital syphilis (CS), in EU/EEA is coordinated by the European Centre for Disease Prevention and Control. Data reported by Member States covering the period 2006-2011 were analysed. Information on country of birth and probable country of infection were used to identify where HIV transmission might have occurred. Rates of HIV and CS per 100,000 live births as well as rates of HIV and syphilis among women of reproductive age (15-49 years) were used to compare mother-to-child transmission across the EU/EEA.

Results In 2006-2011, 592 cases of HIV-MTCT and 566 CS were reported (rate: $1.8 / 100000$ population and 2.0/100000 respectively). 41393 cases of HIV infection and 18750 cases of syphilis were reported in women of reproductive age (rates: 5.7/100000 and $3.1 / 100000$, respectively). Cases of HIV-MTCT and CS were reported by 20 and 16 countries respectively. Rates ranged between 0.2 and 21.8 for HIV and 0.1 and 39.8 for CS. Rates of HIV-MTCT and HIV in women of reproductive age were higher than the EU/ EEA average in France, Latvia, Portugal and the UK. Similarly, rates of CS and syphilis were higher than the EU/EEA average in Bulgaria, Latvia, Romania, Lithuania and Slovakia.

Conclusions Surveillance data suggest an unequal performance across EU/EEA in preventing mother-to-child transmission of HIV and syphilis. Understanding of the epidemiological determinants at national level, identification of pregnant women with high HIV and syphilis burden and barriers for access to antenatal care, will inform revision of antenatal screening practices and will allow strengthening of prevention interventions towards key populations.

\section{P5.009 PERFORMANCE OF CERVICAL CANCER SCREENING TESTS IN HIV POSITIVE WOMEN IN AFRICA: INFLUENCE OF CD4 COUNTS}

doi:10.1136/sextrans-2013-051184.1056

'S Delany, ${ }^{2 \mathrm{H}} \mathrm{A}$ Weiss, ${ }^{3} \mathrm{O}$ Lompo, ${ }^{4} \mathrm{~S}$ Doutre, ${ }^{1} \mathrm{~T}$ Omar, ${ }^{2 \mathrm{H}}$ Kelly, ${ }^{3} \mathrm{~S}$ Zan, ${ }^{1} \mathrm{P}$ Michelow, ${ }^{4} \mathrm{~V}$ Costes, ${ }^{2 P}$ Mayaud. 'Wits Reproductive Health and HIV Institute, Johannesburg, South Africa; ' ${ }^{2}$ ondon School of Hygiene \& Tropical Medicine, London, UK; ${ }^{3}$ Centre de Recherche Internationale sur la Sante, Ouagadougou, Burkina Faso; ${ }^{4}$ University of Montpellier 1 \& INSERM U1058, Montpellier, France

Background To evaluate strategies for cervical cancer screening among HIV-positive African women, against histological CIN endpoints.

Abstract P5.009 Table 1 Prevalence of CIN2+, and performance of screening tests singly or in combination by CD4 + count among HIV-positive women in Burkina Faso and South Africa

\begin{tabular}{|c|c|c|c|c|}
\hline & CD4 $<200$ cells $/ \mu \mathrm{L}$ & CD4 200-349 cells $/ \mu \mathrm{L}$ & CD4 $\geq 350$ cells $/ \mu \mathrm{L}$ & P-value for trend \\
\hline \multicolumn{5}{|l|}{ CIN2+ prevalence } \\
\hline $\begin{array}{l}\text { Burkina Faso } \\
\text { ( } \mathrm{N}=511 \text { evaluated women) }\end{array}$ & $7 / 54(13 \%)$ & $4 / 102(4 \%)$ & $19 / 355(5 \%)$ & $P=0.09$ \\
\hline $\begin{array}{l}\text { South Africa } \\
\text { ( } N=438 \text { evaluated women) }\end{array}$ & $21 / 44(48 \%)$ & $35 / 101(35 \%)$ & $69 / 293(24 \%)$ & $P<0.001$ \\
\hline \multicolumn{5}{|c|}{ Sensitivity to detect CIN2+ (N $=155)$} \\
\hline HR-HPV & $100 \%$ & $94 \%$ & $85 \%$ & $P=0.07$ \\
\hline Cytology (LSIL) & $92 \%$ & $94 \%$ & $94 \%$ & $P=0.81$ \\
\hline Cytology (HSIL) & $80 \%$ & $72 \%$ & $57 \%$ & $P=0.03$ \\
\hline VIA/VILI & $68 \%$ & $64 \%$ & $58 \%$ & $P=0.32$ \\
\hline HPV + Cytology & $100 \%$ & $100 \%$ & $98 \%$ & $P=1.00$ \\
\hline HPV + VIA/VILI & $100 \%$ & $100 \%$ & $93 \%$ & $P=1.00$ \\
\hline Cytology + VIA/VILI & $96 \%$ & $97 \%$ & $96 \%$ & $P=0.99$ \\
\hline \multicolumn{5}{|c|}{ Specificity to detect CIN2+ $(N=155)$} \\
\hline HR-HPV & $36 \%$ & $47 \%$ & $63 \%$ & $\mathrm{P}<0.001$ \\
\hline Cytology (LSIL) & $49 \%$ & $50 \%$ & $56 \%$ & $P=0.13$ \\
\hline Cytology (HSIL) & $87 \%$ & $90 \%$ & $91 \%$ & $P=0.30$ \\
\hline VIA/VILI & $77 \%$ & $74 \%$ & $74 \%$ & $P=0.72$ \\
\hline HPV + Cytology (HSIL) & $29 \%$ & $32 \%$ & $41 \%$ & $P=0.02$ \\
\hline HPV + VIAVILI & $30 \%$ & $38 \%$ & $49 \%$ & $P=0.002$ \\
\hline Cytology (HSIL) + VIA/VILI & $42 \%$ & $42 \%$ & $47 \%$ & $P=0.28$ \\
\hline
\end{tabular}

